\title{
Weak-strong uniqueness for fluid-rigid body interaction problem with slip boundary condition
}

\author{
Nikolai V. Chemetov ${ }^{1}$, Šárka Nečasová ${ }^{2}$, Boris Muha ${ }^{3}$ \\ ${ }^{1}$ University of Lisbon \\ nvchemetov@gmail.com \\ 2 Institute of Mathematics, \\ Žitná 25, 11567 Praha 1, Czech Republic \\ matus@math.cas.cz \\ ${ }^{3}$ Department of Mathematics \\ Faculty of Science \\ University of Zagreb, Croatia \\ borism@math.hr
}

\begin{abstract}
We consider a coupled PDE-ODE system describing the motion of the rigid body in a container filled with the incompressible, viscous fluid. The fluid and the rigid body are coupled via Navier's slip boundary condition. We prove that the local in time strong solution is unique in the larger class of weak solutions on the interval of its existence. This is the first weak-strong uniqueness result in the area of fluid-structure interaction with a moving boundary.
\end{abstract}

\section{Introduction}

A solid body motion in a fluid is a widespread phenomenon in nature, being one of the most classical problem of fluid mechanics. The understanding of the correct mathematical description of fluid-structure interaction has several important applications in many branches, such as in civil engineering, aerospace engineering, nuclear engineering, ocean engineering, biomechanics and etc..

As well-known [9] the fluid motion fulfills the Navier-Stokes equations and the solid motion is described by a system of ordinary differential equations of momentum conservation laws. The fluid and solids are coupled through kinematic and dynamic coupling condition. The most usual 
kinematic coupling condition in the literature is the standard no-slip boundary condition: the continuity of velocities of the fluid and the solids at the fluid-body interfaces. Such approach has been investigated by many authors, e.g. [3]-[8], [17, 24] and references within. Despite lot of progress there are only few uniqueness results for weak solutions. The uniqueness of weak solution for the fluid-rigid body system in $2 D$ was proven in [14. The uniqueness of weak solution to $3 D$ NavierStokes equation is a famous open problem (see e.g. [10]). However, it is well known that strong solution (i.e. solution with some extra regularity or integrability) is unique in a larger class of weak solution on interval of its existence [25]. These type of results are called weak-strong uniqueness results. The main result of this paper is to prove a weak-strong uniqueness result for the fluid-rigid body system. We also mention that in [6] the authors studied the motion of rigid body containing a cavity filled with the fluid and proved a weak-strong uniqueness result for that problem.

However, it has been shown in [15], [16], [26] that the non-slip condition exhibits an unrealistic phenomenon: two smooth solids can not touch each other. The non-slip condition prescribes the adherence of fluid particles to the solid boundaries and, as a consequence of a regularity of the fluid velocity, permits the creation of fine boundary layer that does not allow the contact of the solids.

Another method for coupling of the fluid and of the bodies admits the slippage of fluid particles at the boundaries, which is described by Navier's boundary condition. The first step in this direction of the study of Navier's condition was done by Neustupa, Penel [22], [23], who demonstrate that the collision with a wall can occur for a prescribed movement of a solid ball, when the slippage was allowed on both boundaries. We refer for a discussion of Navier's boundary condition to Introduction of [20]. In this last work a local in time existence result was demonstrated for the motion of the fluid and an elastic structure with prescribed Navier's condition on the boundaries. Motivated by these works, in this paper we study coupling via Navier's slip boundary condition. Also we mention the article [12] where a local existence up to collisions of a weak solution for a fluid-solid structure was proved. The existence of a strong solution in $2 D$ case was proven by Wang [29].

The global in time existence of the weak solution was proven in [2] for a mixed case, when Navier's condition was given on the solid boundary and the non-slip condition on the domain boundary. This result admits the collisions of the solid with the domain boundary (for more detailed discussion concerning influence of boundary conditions on the collision see [13]). Recently the local in time existence of the strong solution for the mixed case was demonstrated in [1].

\section{Preliminaries}

We shall investigate the motion of a rigid body inside of a viscous incompressible fluid. The fluid and the body occupy a bounded open domain $\Omega \subset \mathbb{R}^{N}(N=2$ or 3$)$. Let the body be a connected open set $S_{0} \subset \Omega$ at the initial time $t=0$. The fluid fills the domain $F_{0}=\Omega \backslash \overline{S_{0}}$ at $t=0$.

The Cartesian coordinates $\mathbf{y}$ of points of the body at $t=0$ are called the Lagrangian coordinates. The motion of any material point $\mathbf{y}=\left(y_{1}, . ., y_{N}\right)^{T} \in S_{0}$ is described by two functions:

$$
t \rightarrow \mathbf{q}(t) \in \mathbb{R}^{N} \quad \text { and } \quad t \mapsto \mathbb{Q}(t) \in S O(N) \quad \text { for } \quad t \in[0, T],
$$

where $\mathbf{q}=\mathbf{q}(t)$ is the position of the body mass center at a time $t$ and $S O(N)$ is the rotation 
group in $\mathbb{R}^{N}$, i.e. the $\mathbb{Q}=\mathbb{Q}(t)$ is a matrix, satisfying $\mathbb{Q}(t) \mathbb{Q}(t)^{T}=\mathbb{I}, \mathbb{Q}(0)=\mathbb{I}$ with $\mathbb{I}$ being the identity matrix. Therefore, the trajectories of all points of the body are described by a preserving orientation isometry:

$$
\mathbf{B}(t, \mathbf{y})=\mathbf{q}(t)+\mathbb{Q}(t)(\mathbf{y}-\mathbf{q}(0)) \quad \text { for any } \quad \mathbf{y} \in S_{0}
$$

and the body occupies the set:

$$
S(t)=\left\{\mathbf{x} \in \mathbb{R}^{N}: \mathbf{x}=\mathbf{B}(t, \mathbf{y}), \quad \mathbf{y} \in S_{0}\right\}=\mathbf{B}\left(t, S_{0}\right)
$$

at any time $t$. The velocity of the body, called rigid velocity, is defined as:

$$
\frac{d}{d t} \mathbf{B}(t, \mathbf{y})=\mathbf{u}_{s}(t, \mathbf{x})=\mathbf{a}(t)+\mathbb{P}(t)(\mathbf{x}-\mathbf{q}(t)) \quad \text { for all } \quad \mathbf{x} \in S(t)
$$

where $\mathbf{a}=\mathbf{a}(t) \in \mathbb{R}^{N}$ is the translation velocity and $\mathbb{P}=\mathbb{P}(t)$ is the angular velocity. The velocity $\mathbf{u}_{s}$ has to be compatible with $\mathbf{B}$ in the sense:

$$
\frac{d \mathbf{q}}{d t}=\mathbf{a} \quad \text { and } \quad \frac{d \mathbb{Q}}{d t} \mathbb{Q}^{T}=\mathbb{P} \quad \text { in }[0, T]
$$

The angular velocity $\mathbb{P}$ is a skew-symmetric matrix, i.e. there exists a vector $\boldsymbol{\omega}=\boldsymbol{\omega}(t) \in \mathbb{R}^{N}$, such that

$$
\mathbb{P}(t) \mathbf{x}=\boldsymbol{\omega}(t) \times \mathbf{x}, \quad \forall \mathbf{x} \in \mathbb{R}^{N} .
$$

We define the fluid domain as $\Omega_{F}(t)=\Omega \backslash \overline{S(t)}$. Finally, we introduce a slight abuse of notation to denote the non-cylindrical domains:

$$
(0, T) \times \Omega_{F}(t)=\bigcup_{t \in(0, T)}\{t\} \times \Omega_{F}(t), \quad(0, T) \times \partial S(t)=\bigcup_{t \in(0, T)}\{t\} \times \partial S(t) .
$$

We consider the following problem modeling the motion of the rigid body in viscous incompressible fluids.

Find $(\mathbf{u}, p, \mathbf{q}, \mathbb{Q})$ such that

$$
\begin{aligned}
& \left.\begin{array}{l}
\varrho\left(\partial_{t} \mathbf{u}+(\mathbf{u} \cdot \nabla) \mathbf{u}\right)=\operatorname{div}(\mathbb{T}(\mathbf{u}, p)), \\
\operatorname{div} \mathbf{u}=0
\end{array}\right\} \text { in }(0, T) \times \Omega_{F}(t), \\
& \left.\begin{array}{l}
\frac{d^{2}}{d t^{2}} \mathbf{q}=-\int_{\partial S(t)} \mathbb{T}(\mathbf{u}, p) \mathbf{n} d \gamma(\mathbf{x}), \\
\frac{d}{d t}(\mathbb{J} \boldsymbol{\omega})=-\int_{\partial S(t)}(\mathbf{x}-\mathbf{q}(t)) \times \mathbb{T}(\mathbf{u}, p) \mathbf{n} d \gamma(\mathbf{x})
\end{array}\right\} \text { in }(0, T), \\
& \left(\mathbf{u}-\mathbf{u}_{s}\right) \cdot \mathbf{n}=0, \quad \beta\left(\mathbf{u}_{s}-\mathbf{u}\right) \cdot \boldsymbol{\tau}=\mathbb{T}(\mathbf{u}, p) \mathbf{n} \cdot \boldsymbol{\tau} \quad \text { on }(0, T) \times \partial S(t), \\
& \mathbf{u}(0, .)=\mathbf{u}_{0} \quad \text { in } \Omega ; \quad \mathbf{q}(0)=\mathbf{q}_{0}, \quad \mathbf{q}^{\prime}(0)=\mathbf{a}_{0}, \quad \boldsymbol{\omega}(0)=\boldsymbol{\omega}_{0},
\end{aligned}
$$

where $\mathbf{n}(x)$ is the unit interior normal at $\mathbf{x} \in \partial S(t)$, i.e. the vector $\mathbf{n}$ is directed inside of $S(t), \varrho$ is a constant density of fluid, and $\beta>0$ a constant friction coefficient of $\partial S$. The surface measure 
over a moving surface $\partial S(t)$ is indicated by $d \gamma . \mathbb{J}$ is the matrix of the inertia moments of the body $S(t)$ related to its mass center, calculated as:

$$
\mathbb{J}=\int_{S(t)} \varrho_{S}(t, \mathbf{x})\left(|\mathbf{x}-\mathbf{q}(t)|^{2} \mathbb{I}-(\mathbf{x}-\mathbf{q}(t)) \otimes(\mathbf{x}-\mathbf{q}(t))\right) d \mathbf{x}
$$

where $\varrho_{S}$ is the rigid body density. In (2.6) $\mathbf{u}$ is the fluid velocity; $\mathbb{T}$ is the stress tensor and $\mathbb{D}$ is the deformation-rate tensor, which are defined as:

$$
\mathbb{T}=-p I+2 \mu \mathbb{D} \mathbf{u} \quad \text { and } \quad \mathbb{D} \mathbf{u}=\frac{1}{2}\left(\nabla \mathbf{u}+(\nabla \mathbf{u})^{T}\right)
$$

with $p$ being the fluid pressure and $\mu>0$ being the constant viscosity of the fluid. For simplicity we assume that the fluid density $\varrho$ and the mass of rigid body equals 1 .

Let us introduce the definition of weak solutions for system (2.6) $-(2.9)$. To begin with we define the space [19, 21]:

$$
V^{0,2}(\Omega)=\left\{\mathbf{v} \in L^{2}(\Omega): \quad \operatorname{div} \mathbf{v}=0 \quad \text { in } \mathcal{D}^{\prime}(\Omega), \quad \mathbf{v} \cdot \mathbf{n}=0 \quad \text { in } H^{-1 / 2}(\partial \Omega)\right\},
$$

where $\mathbf{n}$ is the unit normal to the boundary of $\Omega$. Let $\mathcal{M}(\Omega)$ be the space of bounded Radon measures. Let

$$
B D_{0}(\Omega)=\left\{\mathbf{v} \in L^{1}(\Omega): \mathbb{D} \mathbf{v} \in \mathcal{M}(\Omega), \quad \mathbf{v}=0 \quad \text { on } \partial \Omega\right\}
$$

be the space of functions of bounded deformation. Let $S$ be an open connected subset of $\Omega$ with the boundary $\partial S \in C^{2}$. We introduce the following space of vector functions:

$$
\begin{aligned}
& K B(S)=\left\{\mathbf{v} \in B D_{0}(\Omega): \mathbb{D} \mathbf{v} \in L^{2}(\Omega \backslash \bar{S}), \quad \mathbb{D} \mathbf{v}=0 \text { a.e. on } S,\right. \\
& \left.\operatorname{div} \mathbf{v}=0 \text { in } \mathcal{D}^{\prime}(\Omega)\right\} \text {. }
\end{aligned}
$$

Now, we can give a definition of the weak solutions of (2.6) $-(2.9)$.

Definition 2.1 The triple $\{\mathbf{B}, \mathbf{u}\}$ is a weak solution of system (2.6)-(2.9), if the following conditions are satisfied:

1) The function $\mathbf{B}(t, \cdot): \mathbb{R}^{N} \rightarrow \mathbb{R}^{N}$ is a preserving orientation isometry (2.1), which defines a time dependent set $S(t)$ by (2.2). The isometry $\mathbf{B}$ is compatible with $\mathbf{u}=\mathbf{u}_{s}$ on $S(t)$ : the functions $\mathbf{q}, \mathbb{Q}$ are absolutely continuous on $[0, T]$ and satisfy equalities (2.3) $-(2.5)$;

2) The function $\mathbf{u} \in L^{2}(0, T ; K B(S(t))) \cap L^{\infty}\left(0, T ; V^{0,2}(\Omega)\right)$ satisfies the integral equality:

$$
\begin{aligned}
\int_{0}^{T} d t \int_{\Omega \backslash \partial S(t)}\left\{\mathbf{u} \cdot \partial_{t} \boldsymbol{\psi}\right. & \left.+(\mathbf{u} \otimes \mathbf{u}): \mathbb{D} \boldsymbol{\psi}-2 \mu_{f} \mathbb{D} \mathbf{u}: \mathbb{D} \boldsymbol{\psi}\right\} d \mathbf{x} \\
& =-\int_{\Omega} \mathbf{u}_{0} \cdot \boldsymbol{\psi}(0, \cdot) d \mathbf{x}+\int_{0}^{T} d t \int_{\partial S(t)} \beta\left(\mathbf{u}_{s}-\mathbf{u}_{f}\right) \cdot\left(\boldsymbol{\psi}_{s}-\boldsymbol{\psi}_{f}\right) d \gamma
\end{aligned}
$$


which holds for any test function $\boldsymbol{\psi}$, such that

$$
\begin{aligned}
\boldsymbol{\psi} & \in L^{2(N-1)}(0, T ; K B(S(t))), \\
\partial_{t} \boldsymbol{\psi} & \in L^{2}\left(0, T ; L^{2}(\Omega \backslash \partial S(t))\right), \quad \boldsymbol{\psi}(T, \cdot)=0 .
\end{aligned}
$$

By $\mathbf{u}_{s}(t, \cdot), \boldsymbol{\psi}_{s}(t, \cdot)$ and $\mathbf{u}_{f}(t, \cdot), \boldsymbol{\psi}_{f}(t, \cdot)$ we denote the trace values of $\mathbf{u}, \boldsymbol{\psi}$ on $\partial S(t)$ from the "rigid" side $S(t)$ and the "fluid" side $F(t)$, respectively.

Let us recall the global solvability result proved in [2].

Theorem 2.1 Let the boundaries be $\partial \Omega \in C^{0,1}, \partial S_{0} \in C^{2}$. Let us assume that $\overline{S_{0}} \subset \Omega$ and

$$
\mathbf{u}_{0} \in V^{0,2}(\Omega), \quad \mathbb{D} \mathbf{u}_{0}=0 \quad \text { in } \mathcal{D}^{\prime}\left(S_{0}\right) .
$$

Then problem (2.6)-(2.9) possesses a weak solution $\{\mathbf{B}, \mathbf{u}\}$, such that the isometry $\mathbf{B}(t, \cdot)$ is Lipschitz continuous with respect to $t \in[0, T]$,

$$
\mathbf{u} \in C_{\text {weak }}\left(0, T ; V^{0,2}(\Omega)\right) \cap L^{2}(0, T ; K B(S(t)))
$$

and for a.a. $t \in(0, T)$ the following inequality holds:

$$
\frac{1}{2} \int_{\Omega}|\mathbf{u}|^{2}(t) d \mathbf{x}+\int_{0}^{t} d r\left\{\int_{\Omega_{F}(r)} 2 \mu|\mathbb{D} \mathbf{u}|^{2} d \mathbf{x}+\int_{\partial S(r)} \beta\left|\mathbf{u}-\mathbf{u}_{s}\right|^{2} d \gamma\right\} \leq \frac{1}{2} \int_{\Omega}\left|\mathbf{u}_{0}\right|^{2} d \mathbf{x} .
$$

Let us introduce on the fluid domain $\Omega_{F}(t)$ for $t \in(0, T)$ the following function spaces:

$$
L^{2}\left(\Omega_{F}^{1}(t)\right), \quad L^{\infty}\left(\Omega_{F}^{1}(t)\right), \quad W^{1, \infty}\left(\Omega_{F}^{1}(t)\right) \quad \text { for a.e. } t \in(0, T)
$$

and

$$
L^{2}\left(0, T ; L^{2}\left(\Omega_{F}(t)\right)\right), \quad L^{2}\left(0, T ; H^{k}\left(\Omega_{F}(t)\right)\right), \quad H^{k}\left(0, T ; L^{2}\left(\Omega_{F}(t)\right)\right) \quad \text { with } k=1 \text { or } 2 .
$$

Also we recall the local existence result for the strong solution obtained in [1].

Theorem 2.2 Let the boundaries be $\partial \Omega, \partial S_{0} \in C^{2}$. Suppose that $\overline{S_{0}} \subset \Omega$ and

$$
\mathbf{u}_{0} \in K B\left(S_{0}\right) .
$$

Then problem (2.6)-(2.9) possesses a local-in-time strong solution $(\mathbf{B}, \mathbf{u})$, such that the isometry $\mathbf{B}(t, \cdot)$ is Lipschitz continuous with respect to $t \in[0, T]$, and there exists a maximal $T_{0}>0$, such that (2.6)-(2.9) has a unique strong solution $(\mathbf{u}, p, \mathbf{a}(t), \boldsymbol{\omega}(t))$, which for all $T<T_{0}$ satisfies the following energy inequality:

$$
\|\mathbf{u}\|_{L^{2}\left(0, T ; H^{2}\left(\Omega_{F}(t)\right)\right.}+\|p\|_{L^{2}\left(0, T ; H^{1}\left(\Omega_{F}(t)\right)\right.}+\|\mathbf{a}\|_{H^{1}(0, T)}+\|\boldsymbol{\omega}\|_{H^{1}(0, T)} \leq C .
$$

Here and below we denote by $C$ generic constants depending only on the data of our problem (2.6) (2.9). 
The aim of our paper is to prove the weak-strong uniqueness result for system (2.6) -(2.9). More precisely, we prove that on the interval $\left(0, T_{0}\right)$ where the strong solution exists, the strong solution is unique in the class of weak solutions given by Definition 2.1. To the best of our knowledge this is the first weak-strong uniqueness in the area of fluid-structure interaction. Uniqueness of weak solutions in $2 D$ case for the fluid-rigid body system with no-slip coupling condition was proved in [14.

Let us end this section by a well-known Reynolds transport theorem from the fluid mechanics theory, which will be often used in our calculations on moving domains.

Lemma 2.1 Let $V(t)$ be a time dependent volume moved by a smooth divergence-free velocity $\mathbf{v}=$ $\mathbf{v}(t, \mathbf{x})$. Then

$$
\frac{d}{d t} \int_{V(t)} f(t, \mathbf{x}) d x=\int_{V(t)} \dot{f} d \mathbf{x}
$$

for any smooth function $f=f(t, \mathbf{x})$. Here $\dot{f}=\frac{\partial f}{\partial t}+(\mathbf{v} \cdot \nabla) f$ is the total time derivative.

\section{Weak-strong uniqueness}

Let $\left(\mathbf{u}_{1}, \mathbf{a}_{1}, \boldsymbol{\omega}_{1}\right)$ be the triplet consisting of the fluid velocity, the translation rigid body velocity and the angular rigid body velocity connected to the weak solution $\left(\mathbf{u}_{1}, \mathbf{B}_{1}\right)$ (see Definition 2.1), i.e.

$$
\mathbf{B}_{1}(t, \mathbf{y})=\mathbf{q}_{1}(t)+\mathbb{Q}_{1}(t)\left(\mathbf{y}-\mathbf{q}_{0}\right),
$$

where $\mathbf{a}_{1}=\mathbf{q}_{1}^{\prime}(t) \in \mathbb{R}^{3}$ and the vector $\boldsymbol{\omega}_{1}=\boldsymbol{\omega}_{1}(t) \in \mathbb{R}^{3}$ is associated with the skew-symmetric matrix $\mathbb{P}_{1}=\mathbb{Q}_{1}^{\prime} \mathbb{Q}_{1}^{T}$, satisfying the property:

$$
\boldsymbol{\omega}_{1}(t) \times \mathbf{x}=\mathbb{P}_{1}(t) \mathbf{x}, \quad \forall \mathbf{x} \in \mathbb{R}^{3} .
$$

We denote the domain of the rigid body at the time $t$ by

$$
S_{1}(t)=\mathbf{B}_{1}\left(t, S_{0}\right)
$$

and the corresponding fluid domain by

$$
\Omega_{F}^{1}(t)=\Omega \backslash \overline{S_{1}(t)}
$$

Moreover, let $\left(\mathbf{u}_{2}, \mathbf{a}_{2}, \boldsymbol{\omega}_{2}\right)$ be the strong solution given by Theorem 2.2 with the corresponding rigid deformation $\mathbf{B}_{2}$ :

$$
\mathbf{B}_{2}(t, \mathbf{y})=\mathbf{q}_{2}(t)+\mathbb{Q}_{2}(t)\left(\mathbf{y}-\mathbf{q}_{0}\right),
$$


where $\mathbf{a}_{2}=\mathbf{q}_{2}^{\prime}$ and the vector $\boldsymbol{\omega}_{2}$ is associated with the skew-symmetric matrix $\mathbb{P}_{2}=\mathbb{Q}_{2}^{\prime} \mathbb{Q}_{2}^{T}$, satisfying

$$
\boldsymbol{\omega}_{2}(t) \times \mathbf{x}=\mathbb{P}_{2}(t) \mathbf{x}, \quad \forall \mathbf{x} \in \mathbb{R}^{3} .
$$

Also as before, we denote the domain of the rigid body at the time $t$ by

$$
S_{2}(t)=\mathbf{B}_{2}\left(t, S_{0}\right)
$$

and the corresponding fluid domain by

$$
\Omega_{F}^{2}(t)=\Omega \backslash \overline{S_{2}(t)}
$$

In this article our main objective is to demonstrate the following weak-strong uniqueness theorem.

Theorem 3.1 We will prove the weak-strong uniqueness result, i.e.

$$
\left(\mathbf{u}_{1}, \mathbf{a}_{1}, \boldsymbol{\omega}_{1}\right)=\left(\mathbf{u}_{2}, \mathbf{a}_{2}, \boldsymbol{\omega}_{2}\right) \quad \text { on the time interval }\left(0, T_{0}\right) \text {, }
$$

where the strong solution $\left(\mathbf{u}_{2}, \mathbf{a}_{2}, \boldsymbol{\omega}_{2}\right)$ exists.

The demonstration of this theorem we divide on few steps, proving auxiliary Lemmas 3.1f3.4. The major difficulty in the study of this uniqueness result consists from the fact that the fluid domains of $\mathbf{u}_{1}$ and $\mathbf{u}_{2}$ are a priori different and therefore we need to transform the strong solution to the fluid domain of the weak solution in order to compare them.

Let $\mathbf{X}_{1}$ and $\mathbf{X}_{2}$ be two time dependent changes of variables defined in Appendix 4 . Furthermore we define the inverse transform of $\mathbf{X}_{2}$, i.e.

$$
\mathbf{Y}_{2}(t, \cdot)=\mathbf{X}_{2}(t, \cdot)^{-1}
$$

It is easy to see

$$
\mathbf{Y}_{2}\left(t, \mathbf{x}_{2}\right)=\mathbf{q}_{0}+\mathbb{Q}_{2}^{T}(t)\left(\mathbf{x}_{2}-\mathbf{q}_{2}(t)\right), \quad \mathbf{x}_{2} \in \partial S_{2}(t) .
$$

Finally we define the transformation $\widetilde{\mathbf{X}}_{1}: \Omega_{F}^{2}(t) \rightarrow \Omega_{F}^{1}(t)$ in the following way:

$$
\widetilde{\mathbf{X}}_{1}\left(t, \mathbf{x}_{2}\right)=\mathbf{X}_{1}\left(t, \mathbf{Y}_{2}\left(t, \mathbf{x}_{2}\right)\right)
$$

and let $\widetilde{\mathbf{X}}_{2}\left(t, \mathbf{x}_{1}\right)$ be its inverse, i.e.

$$
\widetilde{\mathbf{X}}_{2}(t, \cdot)=\widetilde{\mathbf{X}}_{1}(t, \cdot)^{-1} .
$$

In the neighborhoods of $S_{1}(t)$ and $S_{2}(t)$ the transformations $\widetilde{\mathbf{X}}_{2}$ and $\widetilde{\mathbf{X}}_{1}$ are rigid. They are given with the following expressions:

$$
\left\{\begin{array}{l}
\widetilde{\mathbf{X}}_{1}\left(t, \mathbf{x}_{2}\right)=\mathbf{q}_{1}(t)+\mathbb{Q}_{1}(t) \mathbb{Q}_{2}^{T}(t)\left(\mathbf{x}_{2}-\mathbf{q}_{2}(t)\right) \text { in the neighborhood of } S_{2}(t), \\
\widetilde{\mathbf{X}}_{2}\left(t, \mathbf{x}_{1}\right)=\mathbf{q}_{2}(t)+\mathbb{Q}_{2}(t) \mathbb{Q}_{1}^{T}(t)\left(\mathbf{x}_{1}-\mathbf{q}_{1}(t)\right) \text { in the neighborhood of } S_{1}(t) .
\end{array}\right.
$$


Furthermore we put $\mathbb{Q}=\mathbb{Q}_{2} \mathbb{Q}_{1}^{T}$. Now we define a transformed solution of the strong solution $\left(\mathbf{u}_{2}, p_{2}, \mathbf{a}_{2}, \boldsymbol{\omega}_{2}\right)$, where $p_{2}$ is a respective pressure (see Appendix 4):

$$
\left\{\begin{array}{l}
\mathbf{U}_{2}\left(t, \mathbf{x}_{1}\right)=\mathcal{J}_{\widetilde{\mathbf{X}}_{1}\left(t, \widetilde{\mathbf{X}}_{2}\left(t, \mathbf{x}_{1}\right)\right)} \mathbf{u}_{2}\left(t, \widetilde{\mathbf{X}}_{2}\left(t, \mathbf{x}_{1}\right)\right), \quad P_{2}\left(t, \mathbf{x}_{1}\right)=p_{2}\left(t, \widetilde{\mathbf{X}}_{2}\left(t, \mathbf{x}_{1}\right)\right), \\
\mathbf{A}_{2}(t)=\mathbb{Q}^{T}(t) \mathbf{a}_{2}(t), \quad \boldsymbol{\Omega}_{2}(t)=\mathbb{Q}^{T}(t) \boldsymbol{\omega}_{2}(t)
\end{array}\right.
$$

with $\mathcal{J}_{\widetilde{\mathbf{X}}_{1}\left(t, \widetilde{\mathbf{X}}_{2}\left(t, \mathbf{x}_{1}\right)\right)}=\frac{\partial \widetilde{\mathbf{X}}_{1_{i}}}{\partial \mathbf{x}_{2_{j}}}$. Using formulas $(\underline{3.3)})$ we see that the following equalities hold (see also [11]):

$$
\mathbf{n}_{1}=\mathbb{Q}^{T} \mathbf{n}_{2}, \quad \mathbb{T}\left(\mathbf{u}_{2}, p_{2}\right) \mathbf{n}_{2}=\mathbb{Q} \mathcal{T}\left(\mathbf{U}_{2}, P_{2}\right) \mathbf{n}_{1},
$$

where the transformed stress tensor $\mathcal{T}$ is defined in Appendix. Let us compute how the slip boundary condition is transformed, using the transformed solution (3.4). Let $\mathbf{u}_{s}^{1}, \mathbf{u}_{s}^{2}$ be the velocity of the bodies $S_{1}(t)$ and $S_{2}(t)$, respectively (see (2.3) $)$. We define the transformed rigid velocity:

$$
\mathbf{U}_{s}^{2}\left(t, \mathbf{x}_{1}\right)=\mathbb{Q}^{T} \mathbf{u}_{s}^{2}\left(t, \widetilde{\mathbf{X}}_{2}\left(t, \mathbf{x}_{1}\right)\right)=\mathbf{A}_{2}(t)+\Omega_{2}(t) \times\left(\mathbf{x}_{1}-\mathbf{q}_{1}(t)\right) .
$$

We use (3.5) to verify that $\mathbf{U}_{2}$ satisfies the slip boundary condition:

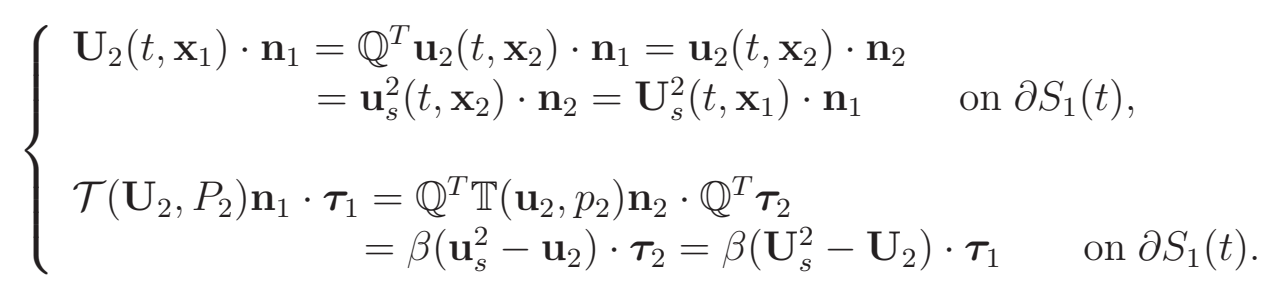

Now we can prove the following lemma.

Lemma 3.1 The transformed solution $\left(\mathbf{U}_{2}, P_{2}, \mathbf{A}_{2}, \Omega_{2}\right)$ of the strong solution $\left(\mathbf{u}_{2}, p_{2}, \mathbf{a}_{2}, \boldsymbol{\omega}_{2}\right)$, defined by (3.4), satisfy the following system of equations on the fluid domain $\Omega_{F}^{1}(t)$ :

$$
\begin{gathered}
\left.\begin{array}{rl}
\partial_{t} \mathbf{U}_{2}+\left(\mathbf{U}_{2} \cdot \nabla\right) \mathbf{U}_{2}-\mu \triangle \mathbf{U}_{2}+\nabla P_{2}=\mu(\mathcal{L}-\triangle) \mathbf{U}_{2}-\mathcal{M} \mathbf{U}_{2} \\
-\tilde{\mathcal{N}} \mathbf{U}_{2}-(G-\nabla) P_{2},
\end{array}\right\} \text { in }(0, T) \times \Omega_{F}^{1}(t), \\
\operatorname{div} \mathbf{U}_{2}=0 \\
\left.\begin{array}{r}
\left(\mathbf{U}_{2}-\mathbf{U}_{s}^{2}\right) \cdot \mathbf{n}_{1}=0, \\
\mathcal{T}\left(\mathbf{U}_{2}, P_{2}\right) \mathbf{n}_{1} \cdot \boldsymbol{\tau}_{1}=\beta\left(\mathbf{U}_{s}^{2}-\mathbf{U}_{2}\right) \cdot \boldsymbol{\tau}_{1}
\end{array}\right\} \text { on }(0, T) \times \partial S_{1}(t), \\
\mathbf{A}_{2}^{\prime}=-\widetilde{\boldsymbol{\omega}} \times \mathbf{A}_{2}-\int_{\partial S_{1}(t)} \mathcal{T}\left(\mathbf{U}_{2}, P_{2}\right) \mathbf{n}_{1} d \gamma\left(\mathbf{x}_{1}\right) \quad \text { in }(0, T), \\
\left(J_{1} \mathbf{\Omega}_{2}\right)^{\prime}=-\widetilde{\boldsymbol{\omega}} \times\left(J_{1} \Omega_{2}\right)-\int_{\partial S_{1}(t)}\left\{\left(\mathbf{x}_{1}-\mathbf{q}_{1}(t)\right) \times \mathcal{T}\left(\mathbf{U}_{2}, P_{2}\right) \mathbf{n}_{1}\right\} d \gamma\left(\mathbf{x}_{1}\right) \quad \text { in }(0, T),
\end{gathered}
$$

where the matrix $J_{1}$ and the vector $\widetilde{\boldsymbol{\omega}}$ are defined by

$$
J_{1}=\mathbb{Q}^{T} J_{2} \mathbb{Q} \quad \text { and } \quad \widetilde{\omega} \times \mathbf{x}=\mathbb{Q}^{T} \mathbb{Q}^{\prime} \mathbf{x},
$$

and the operators $\mathcal{L}, \tilde{\mathcal{N}}, \mathcal{M}$ and $\mathcal{G}$ are defined in the Appendix, (4.1)-(4.4). 
Proof. Equations (3.6) and (3.7) follow from the standard calculations, we refer to Appendix 4 and the above considerations. The interested reader can find complete details of these calculations, for example, in the articles [18, 11]. Let us prove (3.8). We calculate:

$$
\begin{aligned}
\mathbf{A}_{2}^{\prime} & =\left(\mathbb{Q}^{T} \mathbf{a}_{2}\right)^{\prime}=\mathbb{Q}^{T} \mathbf{a}_{2}^{\prime}+\left(\mathbb{Q}^{T}\right)^{\prime} \mathbf{a}_{2}=-\int_{\partial S_{1}(t)} \mathcal{T}\left(\mathbf{U}_{2}, P_{2}\right) \mathbf{n}_{1} d \gamma-\mathbb{Q}^{T} \mathbb{Q}^{\prime} \mathbb{Q}^{T} \mathbf{a}_{2} \\
& =-\int_{\partial S_{1}(t)} \mathcal{T}\left(\mathbf{U}_{2}, P_{2}\right) \mathbf{n}_{1} d \gamma-\widetilde{\boldsymbol{\omega}} \times \mathbf{A}_{2} .
\end{aligned}
$$

Let us now deduce (3.9). We have:

$$
\begin{aligned}
\mathbb{Q}^{T}\left(J_{2} \boldsymbol{\omega}_{2}\right)^{\prime} & =-\mathbb{Q}^{T} \int_{\partial S_{2}(t)}\left\{\left(\mathbf{x}_{2}-\mathbf{q}_{2}(t)\right) \times \mathcal{T}\left(\mathbf{u}_{2}, p_{2}\right) \mathbf{n}_{2}\right\} d \gamma\left(\mathbf{x}_{2}\right) \\
& =-\int_{\partial S_{1}(t)}\left\{\left(\mathbf{x}_{1}-\mathbf{q}_{1}(t)\right) \times \mathcal{T}\left(\mathbf{U}_{2}, P_{2}\right) \mathbf{n}_{1}\right\} d \gamma\left(\mathbf{x}_{1}\right)
\end{aligned}
$$

and on the other hand:

$$
\begin{aligned}
\mathbb{Q}^{T}\left(J_{2} \boldsymbol{\omega}_{2}\right)^{\prime} & =\mathbb{Q}^{T}\left(\mathbb{Q} J_{1} \mathbb{Q}^{T} \boldsymbol{\omega}_{2}\right)^{\prime} \\
& =\mathbb{Q}^{T} \mathbb{Q}^{\prime} J_{1} \boldsymbol{\Omega}_{2}+\left(J_{1} \boldsymbol{\Omega}_{2}\right)^{\prime}=\widetilde{\boldsymbol{\omega}} \times\left(J_{1} \boldsymbol{\Omega}_{2}\right)+\left(J_{1} \Omega_{2}\right)^{\prime} .
\end{aligned}
$$

Therefore combining these last two relations we derive (3.9). Hence this lemma is proven.

As a consequence of the previous Lemma 3.1 we can give the weak formulation for $\left(\mathbf{U}_{2}, P_{2}, \mathbf{A}_{2}, \boldsymbol{\Omega}_{2}\right)$.

Corollary 3.1 Let us denote by

$$
\mathbf{F}=\left\{\begin{array}{lr}
\mu(\mathcal{L}-\triangle) \mathbf{U}_{2}-\mathcal{M} \mathbf{U}_{2}-\tilde{\mathcal{N}} \mathbf{U}_{2}-(\mathcal{G}-\nabla) P_{2} & \text { in }(0, T) \times \Omega_{F}^{1}(t), \\
0 & \text { in }(0, T) \times S_{1}(t) .
\end{array}\right.
$$

Then the transformed solution $\left(\mathbf{U}_{2}, P_{2}, \mathbf{A}_{2}, \mathbf{\Omega}_{2}\right)$ satisfies the following equality:

$$
\begin{aligned}
& \int_{0}^{T} d t \int_{\Omega \backslash \partial S_{1}(t)} \mathbf{U}_{2} \cdot \partial_{t} \boldsymbol{\psi} d \mathbf{x}_{1}+\int_{0}^{T} d t \int_{\Omega_{F}^{1}(t)}\left(\left(\mathbf{u}_{1} \otimes \mathbf{U}_{2}\right): \nabla^{T} \boldsymbol{\psi}-\left(\mathbf{U}_{2}-\mathbf{u}_{1}\right) \cdot \nabla \mathbf{U}_{2} \cdot \boldsymbol{\psi}\right) d \mathbf{x}_{1} \\
& -2 \mu_{f} \int_{0}^{T} d t \int_{\Omega \backslash \partial S_{1}(t)}\left(\mathbb{D} \mathbf{U}_{2}: \mathbb{D} \boldsymbol{\psi}+\mathbf{F} \cdot \boldsymbol{\psi}\right) d \mathbf{x}_{1}=\int_{0}^{T} d t\left\{\int_{\partial S_{1}(t)} \beta\left(\mathbf{U}_{s}^{2}-\mathbf{U}_{2}\right) \cdot\left(\boldsymbol{\psi}_{s}-\boldsymbol{\psi}_{f}\right) d \gamma\left(\mathbf{x}_{1}\right)\right\} \\
& -\int_{\Omega} \mathbf{u}_{0} \boldsymbol{\psi}(0, \cdot) d \mathbf{x}_{1}+\int_{0}^{T}\left(\widetilde{\boldsymbol{\omega}} \times\left(J_{1} \Omega_{2}\right) \cdot \boldsymbol{\psi}_{\omega}+\widetilde{\boldsymbol{\omega}} \times \mathbf{A}_{2} \cdot \boldsymbol{\psi}_{h}\right) d t
\end{aligned}
$$

which holds for any test function $\boldsymbol{\psi}$ satisfying (2.11). Let us note that this function $\boldsymbol{\psi}$ is a rigid one on $S_{1}(t)$, that is

$$
\boldsymbol{\psi}(t, \mathbf{x})=\boldsymbol{\psi}_{h}(t)+\boldsymbol{\psi}_{\omega} \times\left(\mathbf{x}-\mathbf{q}_{1}(t)\right) \quad \text { for } \mathbf{x} \in S_{1}(t)
$$


Proof. Using the Reynolds transport theorem (2.12) we can write the inertial term as:

$$
\begin{aligned}
& \int_{0}^{T} d t \int_{\Omega_{F}^{1}(t)}\left(\partial_{t} \mathbf{U}_{2} \cdot \boldsymbol{\psi}+\mathbf{U}_{2} \cdot \nabla \mathbf{U}_{2} \cdot \boldsymbol{\psi}\right) d \mathbf{x}_{1} \\
= & -\int_{0}^{T} d t \int_{\Omega_{F}^{1}(t)}\left(\mathbf{U}_{2} \cdot \partial_{t} \boldsymbol{\psi}+\mathbf{u}_{1} \cdot \nabla\left(\mathbf{U}_{2} \cdot \boldsymbol{\psi}\right)-\mathbf{U}_{2} \cdot \nabla \mathbf{U}_{2} \cdot \boldsymbol{\psi}\right) d \mathbf{x}_{1}-\int_{\Omega} \mathbf{u}_{0} \boldsymbol{\psi}(0, \cdot) d \mathbf{x}_{1} \\
= & -\int_{0}^{T} d t \int_{\Omega_{F}^{1}(t)}\left(\mathbf{U}_{2} \cdot \partial_{t} \boldsymbol{\psi}+\mathbf{u}_{1} \otimes \mathbf{U}_{2}: \nabla^{T} \boldsymbol{\psi}+\left(\mathbf{u}_{1}-\mathbf{U}_{2}\right) \cdot \nabla \mathbf{U}_{2} \cdot \boldsymbol{\psi}\right) d \mathbf{x}_{1}-\int_{\Omega} \mathbf{u}_{0} \boldsymbol{\psi}(0, \cdot) d \mathbf{x}_{1}
\end{aligned}
$$

The rest of the proof follows directly from Lemma 3.1 in a classical way. The details can be found in Appendix A.1. of the article [2].

Before proceeding with the proof we need to prove the following two Lemmas that give us estimate for the additional terms arriving from transformation in (3.6)-(3.9).

Lemma 3.2 For the vector $\widetilde{\boldsymbol{\omega}}$ defined by (3.10) the equality holds:

$$
\widetilde{\boldsymbol{\omega}}(t)=\Omega_{2}(t)-\boldsymbol{\omega}_{1}(t), \quad t \in\left[0, T_{0}\right]
$$

Proof. Let us denote by $\mathbb{P}_{\Omega_{2}}(t)$ matrix such that $\mathbb{P}_{\Omega_{2}}(t) \mathbf{x}=\Omega_{2}(t) \times \mathbf{x}, \mathbf{x} \in \mathbb{R}^{3}$. Since $\mathbb{Q}(t) \in S O(3)$, $t \in(0, T)$, we have:

$$
\Omega_{2} \times \mathbf{x}=\left(\mathbb{Q}^{T} \boldsymbol{\omega}_{2}\right) \times \mathbf{x}=\mathbb{Q}^{T}\left(\boldsymbol{\omega}_{2} \times \mathbb{Q} \mathbf{x}\right)=\mathbb{Q}^{T} \mathbb{P}_{2} \mathbb{Q} \mathbf{x}
$$

Therefore,

$$
\mathbb{P}_{\Omega_{2}}(t)=\mathbb{Q}^{T}(t) \mathbb{P}_{2}(t) \mathbb{Q}(t)=\mathbb{Q}(t) \mathbb{Q}_{2}^{\prime}(t) \mathbb{Q}_{1}(t)^{T}
$$

Here we used definitions of $\mathbb{Q}$ and $\mathbb{P}_{2}$, i.e. $\mathbb{Q}=\mathbb{Q}_{2} \mathbb{Q}_{1}^{T}, \mathbb{P}_{2}=\mathbb{Q}_{2}^{\prime} \mathbb{Q}_{2}^{T}$. Now, we calculate $\mathbb{Q}^{\prime}$ :

$$
\begin{aligned}
\mathbb{Q}^{\prime} & =\mathbb{Q}_{2}^{\prime} \mathbb{Q}_{1}^{T}+\mathbb{Q}_{2}\left(\mathbb{Q}_{1}^{T}\right)^{\prime}=\mathbb{Q}^{T} \mathbb{Q}_{2}^{\prime} \mathbb{Q}_{1}^{T}-\mathbb{Q}_{2} \mathbb{Q}_{1}^{T} \mathbb{Q}_{1}^{\prime} \mathbb{Q}_{1}^{T} \\
& =\mathbb{Q}\left(\mathbb{Q}^{T} \mathbb{Q}_{2}^{\prime}-\mathbb{Q}_{1}^{\prime}\right) \mathbb{Q}_{1}^{T}=\mathbb{Q}\left(\mathbb{P}_{\Omega_{2}}-\mathbb{P}_{1}\right) .
\end{aligned}
$$

Hence,

$$
\mathbb{P}_{\tilde{\omega}}=\mathbb{Q}^{T} \mathbb{Q}^{\prime}=\left(\mathbb{P}_{\Omega_{2}}-\mathbb{P}_{1}\right)
$$

which finishes the proof of the Lemma.

Lemma 3.3 The following estimate holds:

$$
\begin{aligned}
\| \mu(\mathcal{L}-\triangle) \mathbf{U}_{2} & -\mathcal{M} \mathbf{U}_{2}-\tilde{\mathcal{N}} \mathbf{U}_{2}-(\mathcal{G}-\nabla) P_{2} \|_{L^{2}\left(0, T_{0} ; L^{2}\left(\Omega_{F}^{1}(t)\right)\right)} \\
& \leq C\left(\left\|\mathbf{a}_{1}-\mathbf{A}_{2}\right\|_{L^{2}\left(0, T_{0}\right)}+\left\|\boldsymbol{\omega}_{1}-\Omega_{2}\right\|_{L^{2}\left(0, T_{0}\right)}\right),
\end{aligned}
$$

where $C$ depends only on $\left\|\mathbf{U}_{2}\right\|_{L^{2}\left(0, T_{0} ; H^{2}\left(\Omega_{F}^{1}(t)\right)\right)}$, $\left\|P_{2}\right\|_{L^{2}\left(0, T_{0} ; H^{1}\left(\Omega_{F}^{1}(t)\right)\right)}$ and $\left\|\mathbf{U}_{2}\right\|_{L^{\infty}\left(0, T_{0} ; H^{1}\left(\Omega_{F}(t)\right)\right)}$. 
Proof. First we estimate transformations $\widetilde{\mathbf{X}}_{2}$ and $\widetilde{\mathbf{X}}_{1}$. Since these transformations are rigid in the neighborhood of the rigid body we have:

$$
\widetilde{\mathbf{X}}_{2}\left(t, \mathbf{x}_{1}\right)=\mathbf{q}_{2}(t)+\mathbb{Q}(t)\left(\mathbf{x}_{1}-\mathbf{q}_{1}(t)\right)
$$

We calculate:

$$
\begin{aligned}
\partial_{t} \widetilde{\mathbf{X}}_{2}\left(t, \mathbf{x}_{1}\right) & =\mathbf{a}_{2}(t)+\mathbb{Q}^{\prime}(t)\left(\mathbf{x}_{1}-\mathbf{q}_{1}(t)\right)-\mathbb{Q}(t) \mathbf{a}_{1}(t) \\
& =\mathbb{Q}(t)\left(\mathbf{A}_{2}(t)-\mathbf{a}_{1}(t)+\mathbb{Q}^{T}(t) \mathbb{Q}^{\prime}(t)\left(\mathbf{x}_{1}-\mathbf{q}_{1}(t)\right)\right) \\
& =\mathbb{Q}(t)\left(\left(\mathbf{A}_{2}(t)-\mathbf{a}_{1}(t)\right)+\left(\mathbf{\Omega}_{2}(t)-\boldsymbol{\omega}_{1}(t)\right) \times\left(\mathbf{x}_{1}-\mathbf{q}_{1}(t)\right) .\right.
\end{aligned}
$$

In the last quality we used Lemma 3.2 . By integration of the last equality we obtain:

$$
\left(\widetilde{\mathbf{X}}_{2}-\mathrm{id}\right)\left(t, \mathbf{x}_{1}\right)=\int_{0}^{t} \mathbb{Q}(r)\left(\left(\mathbf{A}_{2}(r)-\mathbf{a}_{1}(r)\right)+\left(\mathbf{\Omega}_{2}(r)-\boldsymbol{\omega}_{1}(r)\right) \times\left(\mathbf{x}_{1}-\mathbf{q}_{1}(r)\right) d r .\right.
$$

Since $\mathbb{Q}$ and $\mathbf{q}_{1}$ are uniformly bounded we get the following estimates on $\partial S_{1}(t)$ for $t \in\left[0, T_{0}\right]$ :

$$
\begin{aligned}
\left|\widetilde{\mathbf{X}}_{2}(t)-\mathrm{id}\right| & \leq C\left(\left\|\mathbf{a}_{1}-\mathbf{A}_{2}\right\|_{L^{2}\left(0, T_{0}\right)}+\left\|\boldsymbol{\omega}_{1}-\boldsymbol{\Omega}_{2}\right\|_{L^{2}\left(0, T_{0}\right)}\right) \\
\left|\partial_{t} \widetilde{\mathbf{X}}_{2}(t)\right| & \leq C\left(\left|\mathbf{a}_{1}(t)-\mathbf{A}_{2}(t)\right|+\left|\boldsymbol{\omega}_{1}(t)-\boldsymbol{\Omega}_{2}(t)\right|\right) .
\end{aligned}
$$

Using the previous estimates and a standard construction of change of variables connected to the rigid motion $\widetilde{\mathbf{X}}_{2}$ (see the articles [11, 27] or for slightly different point of view we refer to Proposition 1 and Corollary 1 of [14]), one gets the following estimates:

$$
\left.\begin{array}{c}
\left\|\widetilde{\mathbf{X}}_{2}(t, .)-\mathrm{id}\right\|_{W^{2, \infty}\left(\Omega_{F}^{1}(t)\right)} \\
\quad \| C\left(\left\|\mathbf{a}_{1}-\mathbf{A}_{2}\right\|_{L^{2}\left(0, T_{0}\right)}+\left\|\boldsymbol{\omega}_{1}-\Omega_{2}\right\|_{L^{2}\left(0, T_{0}\right)}\right), \\
\left\|\partial_{t} \widetilde{\mathbf{X}}_{2}(t, .)\right\|_{W^{1, \infty}\left(\Omega_{F}^{1}(t)\right)} \leq C\left(\left|\mathbf{a}_{1}(t)-\mathbf{A}_{2}(t)\right|+\left|\boldsymbol{\omega}_{1}(t)-\Omega_{2}(t)\right|\right),
\end{array}\right\} \quad t \in\left[0, T_{0}\right]
$$

Analogous estimates can be derived for $\widetilde{\mathbf{X}}_{1}$.

To finish the proof we use the formulas for the transformed differential operators (4.1)-(4.4). Estimates (3.14) imply:

$$
\begin{aligned}
\left\|g_{i j}(t)-\delta_{i j}\right\|_{W^{1, \infty}\left(\Omega_{F}(t)\right)} & +\left\|g^{i j}(t)-\delta_{i j}\right\|_{W^{1, \infty}\left(\Omega_{F}(t)\right)}+\left\|\Gamma_{i j}^{k}(t)\right\|_{L^{\infty}\left(\Omega_{F}(t)\right)} \\
& \leq C\left(\left|\mathbf{a}_{1}-\mathbf{A}_{2}\right|_{L^{2}\left(0, T_{0}\right)}+\left|\boldsymbol{\omega}_{1}-\Omega_{2}\right|_{L^{2}\left(0, T_{0}\right)}\right), \quad t \in\left[0, T_{0}\right] .
\end{aligned}
$$

The proof of this lemma follows from the fact that

$$
\mathbf{U}_{2} \in L^{2}\left(0, T_{0} ; H^{2}\left(\Omega_{F}^{1}(t)\right)\right) \cap L^{\infty}\left(0, T_{0} ; H^{1}\left(\Omega_{F}(t)\right)\right), \quad P_{2} \in L^{2}\left(0, T_{0} ; H^{1}\left(\Omega_{F}^{1}(t)\right)\right) .
$$

Let us give a principal lemma of our article from which Theorem 3.1 follows. 
Lemma 3.4 We have:

$$
\left(\mathbf{u}_{1}, p_{1}, \mathbf{a}_{1}, \boldsymbol{\omega}_{1}\right)=\left(\mathbf{U}_{2}, P_{2}, \mathbf{A}_{2}, \Omega_{2}\right) \quad \text { and } \quad\left(\mathbf{u}_{1}, \mathbf{B}_{1}\right)=\left(\mathbf{u}_{2}, \mathbf{B}_{2}\right) .
$$

Proof. We begin by presenting formal estimates which are valid with some additional integrability (e.g. $\mathbf{u}_{1} \in L_{t}^{8} L_{x}^{4},[28]$ ). After, we show that the result is valid for any weak solution. First we subtract equality (3.11) for $\left(\mathbf{U}_{2}, P_{2}, \mathbf{A}_{2}, \Omega_{2}\right)$ from equality (2.10) for $\left(\mathbf{u}_{1}, p_{1}, \mathbf{a}_{1}, \boldsymbol{\omega}_{2}\right)$. In the obtained identity for the difference:

$$
(\mathbf{u}, p, \mathbf{a}, \boldsymbol{\omega})=\left(\mathbf{u}_{1}-\mathbf{U}_{2}, p_{1}-P_{2}, \mathbf{a}_{1}-\mathbf{A}_{2}, \boldsymbol{\omega}_{1}-\mathbf{\Omega}_{2}\right)
$$

we can take the test function $\boldsymbol{\psi}(r)=\mathbf{u}\left(1-\operatorname{sgn}_{+}^{\varepsilon}(r-t)\right)$ for any fixed $t \in(0, T)$ and pass on $\varepsilon \rightarrow 0$, that gives the identity:

$$
\begin{aligned}
& \int_{0}^{t} d r \int_{\Omega \backslash \partial S_{1}(r)} \frac{1}{2} \partial_{r}|\mathbf{u}(r)|^{2} d \mathbf{x}+\int_{0}^{t} d r \int_{\Omega_{F}^{1}(r)}\left(\mathbf{u}_{1} \otimes \mathbf{u}: \nabla^{T} \mathbf{u}-\mathbf{u} \cdot \nabla \mathbf{U}_{2} \cdot \mathbf{u}\right) d \mathbf{x} \\
& -\int_{\Omega \backslash \partial S_{1}(t)}|\mathbf{u}(t)|^{2} d \mathbf{x}-2 \mu_{f} \int_{0}^{t} d r \int_{\Omega \backslash \partial S_{1}(r)}\left(|\mathbb{D} \mathbf{u}|^{2}-\mathbf{F} \cdot \mathbf{u}\right) d \mathbf{x} \\
& =\int_{0}^{t} d r \int_{\partial S(r)} \beta\left|\mathbf{u}_{s}-\mathbf{u}\right|^{2} d \gamma-\int_{0}^{t}\left(\widetilde{\boldsymbol{\omega}} \times\left(J_{1} \Omega_{2}\right) \cdot \boldsymbol{\omega}+\widetilde{\boldsymbol{\omega}} \times \mathbf{A}_{2} \cdot \mathbf{a}\right) d r .
\end{aligned}
$$

The main difficulty in the study of this relation is to estimate the difference of the convective terms.

Let us combine the convective terms with the fluid acceleration term, then we have:

$$
\begin{aligned}
\int_{0}^{t} d r \int_{\Omega_{F}^{1}(r)}\left(\frac{1}{2} \partial_{r}|\mathbf{u}(r)|^{2}\right. & \left.+\mathbf{u}_{1} \otimes \mathbf{u}: \nabla \mathbf{u}-\mathbf{u} \cdot \nabla \mathbf{U}_{2} \cdot \mathbf{u}\right) d \mathbf{x}-\int_{\Omega_{F}^{1}(t)}|\mathbf{u}(t)|^{2} d \mathbf{x} \\
& =-\frac{1}{2} \int_{\Omega_{F}^{1}(t)}|\mathbf{u}(t)|^{2} d \mathbf{x}-\int_{0}^{t} d r \int_{\Omega_{F}^{1}(r)} \mathbf{u} \cdot \nabla \mathbf{U}_{2} \cdot \mathbf{u} d \mathbf{x} .
\end{aligned}
$$

By integration by parts the last term in the right hand side of this identity is written as:

$$
\int_{0}^{t} d r \int_{\Omega_{F}^{1}(r)} \mathbf{u} \cdot \nabla \mathbf{U}_{2} \cdot \mathbf{u} d \mathbf{x}=-\int_{0}^{t} d r \int_{\Omega_{F}^{1}(r)} \mathbf{u} \cdot \nabla \mathbf{u} \cdot \mathbf{U}_{2} d \mathbf{x}+\int_{0}^{t} d r \int_{\partial \Omega_{F}^{1}(r)}(\mathbf{u} \cdot \mathbf{n})\left(\mathbf{u} \cdot \mathbf{U}_{2}\right) d \gamma
$$

The first term in the right-hand side of (3.16) can be estimated in the standard way (see e.g. Temam [28] ) by using the interpolation:

$$
\begin{aligned}
\left|\int_{0}^{t} d r \int_{\Omega_{F}^{1}(r)} \mathbf{u} \cdot \nabla \mathbf{u} \cdot \mathbf{U}_{2} d \mathbf{x}\right| & \leq \int_{0}^{t}\|\mathbf{u}\|_{L^{4}}\|\nabla \mathbf{u}\|_{L^{2}}\left\|\mathbf{U}_{2}\right\|_{L^{4}} d r \\
& \leq C \int_{0}^{t}\left(\|\mathbf{u}\|_{L^{2}}^{1 / 4}\|\nabla \mathbf{u}\|_{L^{2}}^{3 / 4}+\|\mathbf{u}\|_{L^{2}}\right)\|\nabla \mathbf{u}\|_{L^{2}}\left\|\mathbf{U}_{2}\right\|_{L^{4}} d r \\
& \leq C \varepsilon \int_{0}^{t}\|\nabla \mathbf{u}\|_{L^{2}}^{2} d r+\frac{C}{\varepsilon} \int_{0}^{t}\|\mathbf{u}\|_{L^{2}}^{2}\left(\left\|\mathbf{U}_{2}\right\|_{L^{4}}^{2}+\left\|\mathbf{U}_{2}\right\|_{L^{4}}^{8}\right) d r .
\end{aligned}
$$


Here we used the notation $\|\cdot\|_{L^{p}}=\|\cdot\|_{L^{p}}\left(\Omega_{F}^{1}(r)\right)$ for $p=2$ or 4 .

The second term in the right-hand side of (3.16) is estimated as follows:

$$
\begin{aligned}
& \left|\int_{0}^{t} d r \int_{\partial \Omega_{F}^{1}(r)}(\mathbf{u} \cdot \mathbf{n})\left(\mathbf{u} \cdot \mathbf{U}_{2}\right) d \gamma\right| \\
\leq & C \int_{0}^{t}(|\mathbf{a}(r)|+|\boldsymbol{\omega}(r)|)\|\mathbf{u}(r)\|_{L^{2}\left(\partial S_{1}(r)\right)}\left\|\mathbf{U}_{2}(r)\right\|_{L^{2}\left(\partial S_{1}(r)\right)} d r \\
\leq & C \int_{0}^{t}(|\mathbf{a}(r)|+|\boldsymbol{\omega}(r)|)\|\mathbb{D} \mathbf{u}(r)\|_{L^{2}\left(\Omega_{F}^{1}(r)\right)}\left\|\mathbb{D} \mathbf{U}_{2}(r)\right\|_{L^{2}\left(\Omega_{F}^{1}(r)\right)} d r \\
\leq & \frac{C}{\varepsilon} \int_{0}^{t}\|\mathbf{u}(r)\|_{L^{2}(\Omega)}^{2}\left\|\mathbb{D} \mathbf{U}_{2}(r)\right\|_{L^{2}\left(\Omega_{F}^{1}(r)\right)}^{2} d r+C \varepsilon \int_{0}^{t}\|\mathbb{D} \mathbf{u}(r)\|_{L^{2}\left(\Omega_{F}^{1}(r)\right)}^{2} d r \\
\leq & \frac{C}{\varepsilon} \int_{0}^{t}\|\mathbf{u}(r)\|_{L^{2}(\Omega)}^{2} d r+C \varepsilon \int_{0}^{t}\|\mathbb{D} \mathbf{u}(r)\|_{L^{2}\left(\Omega_{F}^{1}(r)\right)}^{2} d r .
\end{aligned}
$$

Moreover, applying (2.12) of the Reynolds transport theorem we have:

$$
\frac{1}{2} \frac{d}{d t} \int_{S_{1}(t)}|\mathbf{u}|^{2} d \mathbf{x}=\frac{1}{2} \int_{S_{1}(t)} \partial_{t}|\mathbf{u}|^{2} d \mathbf{x}+\int_{S_{1}(t)} \mathbf{u}_{1} \otimes \mathbf{u}: \nabla \mathbf{u} d \mathbf{x} .
$$

Finally, the remainder terms in (3.15) can be estimated by using Lemmas 3.3 and 3.2 , By putting these estimates together we conclude:

$$
\begin{aligned}
\|\mathbf{u}(t)\|_{L^{2}(\Omega)}^{2} & +2 \mu \int_{0}^{t}\|\mathbb{D} \mathbf{u}(r)\|_{L^{2}\left(\Omega_{F}^{1}(r)\right)}^{2} d r \leq C \varepsilon \int_{0}^{t}\|\mathbb{D} \mathbf{u}(r)\|_{L^{2}\left(\Omega_{F}^{1}(r)\right)}^{2} d r \\
& +C \int_{0}^{t}\left(|\mathbf{a}(r)|^{2}+|\boldsymbol{\omega}(r)|^{2}\right) d r \\
& +\frac{C}{\varepsilon} \int_{0}^{t}\|\mathbf{u}(r)\|_{L^{2}(\Omega)}^{2}\left(\left\|\mathbf{U}_{2}(r)\right\|_{L^{4}\left(\Omega_{F}^{1}(r)\right)}^{2}+\left\|\mathbf{U}_{2}(r)\right\|_{L^{4}\left(\Omega_{F}^{1}(r)\right)}^{8}+1\right) d r \\
& +C \int_{0}^{t}(|\mathbf{a}(r)|+|\boldsymbol{\omega}(r)|)\|\mathbf{u}(r)\|_{L^{2}(\Omega)} d r .
\end{aligned}
$$

Using Young's inequality and taking $\varepsilon=\frac{\mu}{C}$, the term $\|\mathbb{D} \mathbf{u}(r)\|_{L^{2}\left(\Omega_{F}^{1}(r)\right)}^{2}$ can be absorbed in the left-hand side and we get:

$$
\|\mathbf{u}(t)\|_{L^{2}(\Omega)}^{2} \leq C \int_{0}^{t}\|\mathbf{u}(r)\|_{L^{2}(\Omega)}^{2}\left(1+\left\|\mathbf{U}_{2}(r)\right\|_{L^{4}\left(\Omega_{F}^{1}(r)\right)}^{2}+\left\|\mathbf{U}_{2}(r)\right\|_{L^{4}\left(\Omega_{F}^{1}(r)\right)}^{8}\right) d r .
$$

Hence we finish the proof by applying the integral Gronwall's inequality and conclude that $\mathbf{u}=0$.

Justification of formal calculation. Notice that $\mathbf{u}$ does not have enough regularity to justify previous calculations. However, the formal calculation can be justified in the standard way (e.g. see the proof of Theorem 4.2 in [10], or the proof of Theorem III.3.9 in [28]) using the fact that $\mathbf{U}_{2}$ 
is a strong solution with the regularity stated in Theorem 2.2. Namely, $\mathbf{U}_{2}$ can be taken as a test function in the weak formulation for the solution $\mathbf{u}_{1}$. On the other hand, one can multiply (3.6) by $\mathbf{u}_{1}$ and integrate by parts in just space variables. By combining these two equalities together with the energy equality for $\mathbf{U}_{2}$ and the energy inequality for $\mathbf{u}_{1}$ one can recover the presented formal calculation. We emphasize that in this argument we used the strong regularity of $\mathbf{U}_{2}$, and therefore we did not need any regularization.

\section{Appendix. Local transformation}

Since the fluid domain depends on the motion of the rigid body, we transform the problem to a fixed domain. We define the local transformation as in Takahaski [27]. Let us point that such type of transformation firstly was suggested by Inoue and Wakimoto [18] and then extensively used in the context of strong solution to fluid-rigid body systems (see e.g. [11, 27]). Here we just briefly repeat the main facts about this transformation for the convenience of the reader. Let us just emphasize that our case is slightly different since we are not transforming to the fixed cylindrical domain, but form one moving domain to the other. However, the essential fact for this transformation is that the change of variable is volume preserving diffeomorphism - which is true also on our case.

Let us $\delta(t)=\operatorname{dist}(S(t), \partial \Omega)$. We fix $\delta_{0}$ such that $\delta(t)>\delta_{0}$ and define the solenoidal velocity field $\Lambda(t, \mathbf{x})$ such that $\Lambda=0$ in the $\delta_{0} / 4$ neighborhood of $\partial \Omega, \Lambda=\mathbf{a}(t)+\boldsymbol{\omega}(t) \times(\mathbf{x}-\mathbf{q}(t))$ in the $\delta_{0} / 4$ neighborhood of $S(t)$. Let us define the flow $\mathbf{X}(t): \Omega \rightarrow \Omega$ as the unique solution of the system:

$$
\frac{d}{d t} \mathbf{X}(t, \mathbf{y})=\Lambda(t, \mathbf{X}(t, \mathbf{y})), \quad \mathbf{X}(0, \mathbf{y})=\mathbf{y}, \quad \forall \mathbf{y} \in \Omega .
$$

We denote $\mathbf{Y}$ the inverse of $\mathbf{X}$, i.e.

$$
\mathbf{Y}(t, \cdot)=\mathbf{X}(t, \cdot)^{-1}
$$

Let us write the unknown functions $(\mathbf{u}, p, \boldsymbol{\omega}, \mathbf{a}, \mathbb{T})$ by the change of variables $\mathbf{x} \rightarrow \mathbf{y}$. Then in the system of coordinates $\mathbf{y} \in \Omega$ we obtain the new unknown functions:

$$
\begin{aligned}
& \mathbf{U}(t, \mathbf{y})=\mathcal{J}_{Y}(t, \mathbf{X}(t, \mathbf{y})) \mathbf{u}(t, \mathbf{X}(t, \mathbf{y})), \quad P(t, \mathbf{y})=p(t, \mathbf{X}(t, \mathbf{y})), \\
& \begin{array}{l}
\Xi(t)=\mathbb{Q}^{t}(t) \boldsymbol{\omega}(t), \\
\mathcal{T}(\mathbf{U}(t, \mathbf{y}), P(t, \mathbf{y}))=\mathbb{Q}^{T}(t) \mathbb{T}(\mathbb{Q}(t) \mathbf{U}(t, \mathbf{y}), P(t, \mathbf{y})) \mathbb{Q}(t)
\end{array} \quad \text { for } t \in[0, T], \quad \mathbf{y} \in \Omega_{0},
\end{aligned}
$$

The Jacobian of this change of variables $\mathbf{x} \rightarrow \mathbf{y}$ is denoted by

$$
\mathcal{J}_{Y}(t, \mathbf{X}(t, \mathbf{y}))=\left(\frac{\partial Y_{i}}{\partial x_{j}}\right)
$$

In the sequel we derive a system which satisfies the new unknown functions $(\mathbf{U}, P, \Xi, \xi, \mathcal{T})$. Our system (2.6)-(2.9) is written in terms of the variables $(t, \mathbf{x})$, therefore we have to rewrite these equations in terms of the new variables $\mathbf{Y}=\mathbf{Y}(t, \mathbf{x})$. 
Let us first note that the determinant of the Jacobian $\mathcal{J}_{Y}$ equals to 1 , since $\Lambda$ is a divergence free vector field. Hence using these change of variables we have:

$$
\begin{aligned}
\int_{\partial S(t)} \mathbb{T}(\mathbf{u}, p) \mathbf{n}(t) d \gamma(\mathbf{x}) & =\mathbb{Q} \int_{\partial S(0)} \mathcal{T}(\mathbf{U}, P) \mathbf{N} d \sigma(\mathbf{y}), \\
\int_{\partial S(t)}(\mathbf{x}-\mathbf{q}(t)) \times \mathbb{T}(\mathbf{u}, p) \mathbf{n}(t) d \gamma(\mathbf{x}) & =\mathbb{Q} \int_{\partial S(0)} \mathbf{y} \times \mathcal{T}(\mathbf{U}, P) \mathbf{N} d \sigma(\mathbf{y}),
\end{aligned}
$$

where $d \sigma$ indicates the surface measure over non-moving surface $\partial S(0)$.

In the sequel we derive the equations which satisfy these new unknown functions $(\mathbf{U}, P, \Xi, \xi, \mathcal{T})$. Let us introduce the metric covariant tensor

$$
g_{i j}=X_{k, i} X_{k, j}, \quad X_{k, i}=\frac{\partial X_{k}}{\partial y_{i}}
$$

the metric covariant tensor

$$
g^{i j}=Y_{i, k} Y_{j, k}, \quad Y_{i, k}=\frac{\partial Y_{i}}{\partial x_{k}}
$$

and the Christoffel symbol (of the second kind)

$$
\Gamma_{i j}^{k}=\frac{1}{2} g^{k l}\left(g_{i l, j}+g_{j l, i}-g_{i j, l}\right), \quad g_{i l, j}=\frac{\partial g_{i l}}{\partial y_{j}} .
$$

It is easy to observe that in particular it holds:

$$
\Gamma_{i j}^{k}=Y_{k, l} X_{l, i j}, \quad X_{l, i j}=\frac{\partial X_{l}}{\partial y_{i} \partial y_{j}} .
$$

Hence under the change of variables $\mathbf{x} \rightarrow \mathbf{y}$ the operator $\mathcal{L}$ is the transformed Laplace operator and it is given by

$$
\begin{aligned}
(\mathcal{L} \mathbf{u})_{i}= & \sum_{j, k=1}^{n} \partial_{j}\left(g^{j k} \partial_{k} \mathbf{u}_{i}\right)+2 \sum_{j, k, l=1}^{n} g^{k l} \Gamma_{j k}^{i} \partial_{l} \mathbf{u}_{j} \\
& +\sum_{j, k, l=1}^{n}\left(\partial_{k}\left(g^{k l} \Gamma_{j l}^{i}\right)+\sum_{m=1}^{n} g^{k l} \Gamma_{j l}^{m} \Gamma_{k m}^{i}\right) \mathbf{u}_{j} .
\end{aligned}
$$

The convection term is transformed into

$$
(\mathcal{N} \mathbf{u})_{i}=\sum_{j=1}^{n} \mathbf{u}_{j} \partial_{j} \mathbf{u}_{i}+\sum_{j, k+1}^{n} \Gamma_{j k}^{i} \mathbf{u}_{j} \mathbf{u}_{k}=(\mathbf{u} \cdot \nabla \mathbf{u})_{i}+(\tilde{\mathcal{N}} \mathbf{u})_{i}
$$

The transformation of time derivative and gradient are given by

$$
(\mathcal{M} \mathbf{u})_{i}=\sum_{j=1}^{n} \dot{\mathbf{Y}}_{j} \partial_{j} \mathbf{u}_{i}+\sum_{j, k=1}^{n}\left(\Gamma_{j k}^{i} \dot{\mathbf{Y}}_{k}+\left(\partial_{k} \mathbf{Y}_{i}\right)\left(\partial_{j} \dot{\mathbf{X}}_{k}\right)\right) \mathbf{u}_{j}
$$


The gradient of pressure is transform as follows:

$$
(\mathcal{G} p)_{i}=\sum_{j=1}^{n} g^{i j} \partial_{j} p .
$$

Therefore combining all formulas (4.1)-(4.4) we see that after the change of variables the system (2.6) $-(2.9)$ is transformed into the following system:

$$
\left.\begin{array}{rl}
\partial_{t} \mathbf{U}+(\mathcal{M}-\mu \mathcal{L}) \mathbf{U}=-\mathcal{N}(\mathbf{U})-\mathcal{G} p, \\
\operatorname{div} \mathbf{U}=0
\end{array}\right\} \text { in }(0, T) \times \Omega_{F}(0),
$$

where $\mathbf{U}_{s}=\boldsymbol{\Xi}(t) \times \mathbf{y}+\boldsymbol{\xi}(t)$ is the transformed rigid velocity $\mathbf{u}_{s} ; \mathbf{N}=\mathbf{N}(\mathbf{y})$ is the unit normal at $\mathbf{y} \in \partial S(0)$, directed inside of $S(0) ; I=\mathbb{Q}^{t} \mathbb{U} \mathbb{Q}$ is the transformed inertia tensor which no longer depends on time (see details in the article [11]).

\section{Acknowledgements:}

The authors would like to thank the anonymous referee for her/his comments that helped us to improve the manuscript. We also would like to thank $A$. Radošević for discussion and her comments

about the manuscript. The work of $\check{S}$. Nečasová was supported by Grant No. 16-03230S of GA $\check{C}$ in the framework of RVO 67985840. The work of B. Muha was supported by by Croatian Science Foundation grant number $947 \%$.

\section{References}

[1] H. Al Baba, N.V. Chemetov, B. Muha and Š. Nečasová, "Strong solutions in $L^{2}$ framework for fluid-rigid body interaction problem - mixed case conditions," Topological Methods in Nonlinear Analysis (2018).

[2] N. Chemetov and Š. Nečasová, "The motion of the rigid body in the viscous fluid including collisions. Global solvability result," Nonlinear Anal. Real World Appl. 34, 416-445 (2017). 
[3] C. Conca, J. San Martin and M. Tucsnak, "Existence of solutions for the equations modelling the motion of a rigid body in a viscous fluid," Commun. Partial Differential Equations 25, 1019-1042 (2000).

[4] B. Desjardins and M.J. Esteban, "Existence of weak solutions for the motion of rigid bodies in a viscous fluid," Arch. Rational Mech. Anal. 146, 59-71 (1999).

[5] B. Desjardins and M.J. Esteban, "On weak solutions for fluid-rigid structure interaction: Compressible and incompressible models," Commun. Partial Differential Equations 25, 1399-1413 (2000).

[6] K. Disser, G.P. Galdi, G. Mazzone and P. Zunino, "Inertial motions of a rigid body with a cavity filled with a viscous liquid," Arch. Rational Mech. Anal. 221, no. 1, 487-526 (2016).

[7] E. Feireisl, "On the motion of rigid bodies in a viscous compressible fluid," Arch. Rational Mech. Anal. 167, 281-308 (2003).

[8] E. Feireisl, M. Hillairet and Š. Nečasoá, "On the motion of several rigid bodies in an incompressible non-Newtonian fluid," Nonlinearity 21, 1349-1366 (2008).

[9] G.P. Galdi, On the motion of a rigid body in a viscous liquid: A mathematical analysis with applications. Handbook of Mathematical Fluid Dynamics, Volume 1, Ed. by Friedlander, D. Serre (Elsevier 2002).

[10] G.P. Galdi, An introduction to the Navier-Stokes initial-boundary value problem. In the series: Fundamental directions in mathematical fluid mechanics, 1-70 (Springer, 2000).

[11] M. Geissert, K. Götze and M. Hieber, " $L_{p}$-theory for strong solutions to fluid-rigid body interaction in Newtonian and generalized Newtonian fluids," Trans. Amer. Math. Soc. 365, no. $3,1393-1439$ (2013).

[12] D. Gérard-Varet and M. Hillairet, "Existence of weak solutions up to collision for viscous fluid-solid systems with slip," Comm. Pure Appl. Math. 67, no. 12, 2022-2075 (2014).

[13] D. Gérard-Varet, M. Hillairet and C. Wang, "The influence of boundary conditions on the contact problem in a 3D Navier-Stokes flow," J. Math. Pures Appl. 103, no. 1, 1-38 (2015).

[14] O. Glass and F. Sueur, "Uniqueness Results for Weak Solutions of Two-Dimensional FluidSolid Systems," Arch. Rational Mech. Anal. 218, no. 2, 907-944 (2015).

[15] T.I. Hesla, "Collision of smooth bodies in a viscous fluid: A mathematical investigation," PhD Thesis (Minnesota, 2005).

[16] M. Hillairet, "Lack of collision between solid bodies in a 2D incompressible viscous flow," Comm. Partial Differential Equations 32, no. 7-9, 1345-1371 (2007). 
[17] K.-H. Hoffmann and V.N. Starovoitov, "On a motion of a solid body in a viscous fluid. Two dimensional case," Adv. Math. Sci. Appl. 9, 633-648 (1999).

[18] A. Inoue and M. Wakimoto, "On existence of solutions of the Navier-Stokes equation in a time dependent domain," J. Fac. Sci. Univ. Tokyo Sect. IA Math. 24, no. 2, 303-319 (1977).

[19] P.-L. Lions, Mathematical topics in Fluid mechanics. Volume 1: Incompressible models (Claredon Press, Oxford, 1996).

[20] B. Muha and S. Canic, "Existence of a weak solution to a fluid-elastic structure interection problem with the Navier slip boundary condition," J. Diff. Equations 260, no. 12, 8550-8589 (2016).

[21] J. Nečas, Direct methods in the theory of elliptic equations. Translated from the 1967 French original by Gerard Tronel and Alois Kufner. Editorial coordination and preface by Sárka Nečasová and a contribution by C.G. Simader, Springer Monographs in Mathematics (Springer, Heidelberg, 2012).

[22] J. Neustupa and P. Penel, "Existence of a weak solution to the Navier-Stokes equation with Navier's boundary condition around striking bodies," Comptes Rendus Mathematique 347, no. 11-12, 685-690 (2009).

[23] J. Neustupa and P. Penel, "A Weak solvability of the Navier-Stokes equation with Navier's boundary condition around a ball striking the wall," In the book: Adv. in Math. Fluid Mechanics. Dedicated to Giovanni Paolo Galdi, 385-408 (Springer-Verlag Berlin, 2010).

[24] J.A. San Martin, V.N. Starovoitov and M. Tucsnak, "Global weak solutions for the two dimensional motion of several rigid bodies in an incompressible viscous fluid," Arch. Rational Mech. Anal. 161, 93-112, 2002.

[25] J. Serrin, "The initial value problem for the Navier-Stokes equations," In the series: Nonlinear Problems: Proc. Sympos., Madison, Wis., 1962, 69-98 (Univ. of Wisconsin Press, Madison, Wis., 1963).

[26] V.N. Starovoitov, Behavior of a Rigid Body in an Incompressible Viscous Fluid near a Boundary. In: Intern. Series of Numerical Math. 147, 313-327 (Birkhauser Basel, 2003).

[27] T. Takahashi, "Analysis of strong solutions for the equations modeling the motion of a rigidfluid system in a bounded domain," Adv. Differential Equations 8, no. 12, 1499-1532 (2003).

[28] R. Temam, Navier-Stokes Equation: Theory and Numerical Analysis (North-Holland, 1977).

[29] C. Wang, "Strong solutions for the fluid-solid systems in a 2-D domain," Asymptot. Anal. 89, no. 3-4, 263-306 (2014). 